\title{
MAPPING PROPERTIES OF RELATIVELY REGULAR OPERATORS
}

\author{
S. R. CARADUS 1
}

ABSTRACT. A relatively regular operator is one with closed complemented range and nullspace. It is shown that if $T$ is relatively regular and $f$ is univalent on the spectrum of $T$ with $f(0)=0$, then $f(T)$ is also relatively regular.

In [2], Atkinson studied bounded linear operators $T$ on Banach space $X$ with the property that the equation $T S T=T$ has a solution $S$ in $B(X)$; such operators he called relatively regular. It is well known that relatively regular operators are precisely those with closed complemented range and nullspace. In fact, when $T S T=T$, then $T S$ is a projection onto $R(T)$ and $I-S T$ is a projection onto $N(T)$.

1. In this note, we address ourselves to the following question: if $T$ is relatively regular, what can one say about $p(T)$ when $p$ is a polynomial or, more generally, about $f(T)$ when $f$ is a function analytic on the spectrum of $T$ ? At first, one is discouraged by the situation in Hilbert space; all operators with closed range are now relatively regular and examples are known of the following situations:

(i) bounded operator $T$ with closed range such that $T-\lambda I$ does not have closed range for any $\lambda$ satisfying $0<|\lambda| \leq 1$;

(ii) bounded $T$ with closed range such that $T^{2}$ does not have closed range.

The following constructions show how to get such examples:

(i) Let $N$ be any operator on Hilbert space such that $N-\lambda I$ does not have closed range for any $\lambda,|\lambda| \leq 1$; we could, for example, take $L^{2}(D)$, where $D$ is the closed unit disc, and define $N f(t)=t f(t)$. Then define $T \in B(H \oplus H)$ by the equation $T(f, g)=(0, f+N g)$. Then the range of $T$ is

Received by the editors August 9, 1973 and, in revised form, January 22, 1974. AMS (MOS) subject classifications (1970). Primary 47A50, 47B30; Secondary 15A09.

Key words and phrases. Relatively regular operator, operational calculus.

${ }^{1}$ Research partly supported by NCR Operating Grant A3985. 
$0 \oplus H$, whereas if $0<|\lambda| \leq 1$, it is easy to verify that $T-\lambda I$ does not have closed range. (This construction is essentially due to E. Asplund [1].)

(ii) Let $N$ be any operator on Hilbert space such that $N^{2}=0$ and $N$ has closed range. Let $C$ be any compact operator such that $N C N$ is not finite dimensional. Examples in $l^{2}$ could be given by: $N e_{k}=e_{2 k}$ when $k$ is odd, $N e_{k}=0$ when $k$ is even; $C e_{k}=e_{k+1} / k^{2}$, where $\left\{e_{k}\right\}^{\infty}$ is the usual orthonormal basis. Then $N C N e_{k}=e_{4 k+2} / 4 k^{2}$.

Now for each $\lambda$ in the resolvent set of $C$, consider $T=N(\lambda I-C)$. Clearly $T$ has closed range. But $T^{2}=-N C N(\lambda I-C)$ has range equal to the range of $N C N$ and so $T^{2}$ does not have closed range.

It turns out that the most natural hypothesis which excludes the above possibilities gives a positive result. For our proof we need a lemma of Atkinson [2], the proof of which is so simple that we reproduce it here.

Lemma. Suppose that $T$ is a bounded linear operator on Banach space $X$ and that for some bounded linear operator $R, T R T-T$ is relatively regular. Then $T$ is relatively regular.

Proof. Since $T R T-T$ is relatively regular, there exists a bounded operator $S$ such that

$$
(T R T-T) S(T R T-T)=T R T-T .
$$

But this equation can be rearranged to read

$$
T\{R-(R T-I) S(T R-I)\} T=T,
$$

from which the conclusion follows.

We now can prove the main result.

Theorem 1. Let $T$ be a relatively regular operator on Banach space $X$. Let $f$ be a complex-valued function which is analytic and univalent on a Cauchy domain $D$ which contains the spectrum $\sigma(T)$ and $\{0\}$. Then, if $f(0)=0$, the operator $f(T)$, defined by means of the operational calculus, is relatively regular.

Proof. Since $f$ is analytic and univalent on $D$ and $f(0)=0$, we know that $f^{\prime}(0) \neq 0$ and that there exists a function $g$, analytic and univalent on $f(D)$ such that $g(f(\lambda))=\lambda$. Without loss of generality, we may assume $f^{\prime}(0)=g^{\prime}(0)=1$. Now by a well-known property of the operational calculus, we can write $g(f(T))=T$. Moreover, $g(\lambda)$ can be written as $g(\lambda)=\lambda-\lambda^{2} b(\lambda)$ for some function $b$, analytic on $f(D)$. Hence

$$
T=f(T)-[f(T)]^{2} b(f(T))=f(T)-f(T) b(f(T)) f(T)
$$


From the Lemma, we deduce the required result.

Remark. Suppose that $T S T=T$ in the above theorem. Then we know that there exists a bounded operator $\hat{S}$ such that $f(T) \hat{S} f(T)=f(T)$. By the proof of the Lemma, we get an explicit formula for $\hat{S}$ :

$$
\hat{S}=b(f(T))-[b(f(T)) f(T)-I] S[h(f(T)) f(T)-I],
$$

and hence formulas for projections onto the range and nullspace of $f(T)$.

2. The above results can be extended to unbounded operators in a reasonably straightforward manner. In such a case, we will call an operator relatively regular if its range and nullspace are closed and complemented. (The alternative definition could also be used but some care needs to be taken with the domains of definition of the operators involved.) Suppose that $T$ is a closed unbounded relatively regular operator whose resolvent set $\rho(T)$ is nonempty. Let us fix $\alpha \neq 0$ in $\rho(T)$. If $f$ is analytic on an open set $D$ containing $\sigma(T)$ and also at infinity, and if $f$ is univalent on $D$, define

$$
\phi(\lambda)=f(\alpha \lambda /(\lambda-1))
$$

If $D^{\prime}$ is the inverse image of $D$ under the map $\lambda \rightarrow a \lambda /(\lambda-1)$, then it is easy to check that $\phi$ is univalent and analytic on $D^{\prime}$. Moreover, we can define $f(T)$ by, writing

$$
f(T)=\phi\left(T(T-\alpha I)^{-1}\right)
$$

observing that $T(T-a I)^{-1}$ is bounded since it can be written as $I+$ $\alpha(T-\alpha I)^{-1}$. The conventional version [3] of the operational calculus of unbounded operators is obtained by defining

$$
\psi(\lambda)=f(\alpha+1 / \lambda) \text { and } f(T)=\psi\left[(T-\alpha I)^{-1}\right] .
$$

It is not difficult to see that the two versions agree.

Finally, we observe that if $T$ is an unbounded relatively regular operator, then $T(T-\alpha I)^{-1}$ is a bounded relatively regular operator, for both have the same range, and if $P$ is a projection onto the nullspace of $T$, then $(T-a I) P(T-\alpha I)^{-1}$ is a closed operator defined on all of $X$ and is hence continuous; it is clearly a projection onto the nullspace of $T(T-\alpha I)^{-1}$. We can therefore apply Theorem 1 to $\phi$ and $T(T-\alpha I)^{-1}$ and obtain

Theorem 2. Let $T$ be a closed unbounded relatively regular operator with nonempty resolvent set. Let $f$ be analytic and univalent on a Cauchy domain $D$ containing $\sigma(T)$, and let $f$ be analytic at infinity. Then, if $f(0)=$ 0 , the operator $f(T)$ is relatively regular. 
3. The case of polynomials $p(T)$ when $T$ is an unbounded relatively regular operator is not included in the above theorem and my efforts to settle this case have been unsuccessful. By a simple argument, however, we can settle the Hilbert space case. For if $p$ is univalent on $\sigma(T)$ and $p(0)=0$, then all the other zeros of $p$ must lie in the resolvent set of $T$ and hence we can write

$$
p(T)=\left(T-\lambda_{1}\right)\left(T-\lambda_{2}\right) \cdots\left(T-\lambda_{\zeta}\right) T
$$

from which it is easy to deduce that if $T$ has closed range, so does $p(T)$.

\section{REFERENCES}

1. E. Asplund, A non-closed relative spectrum, Ark. Mat. 3 (1958), 425-427. MR 19, 968.

2. F. V. Atkinson, On relatively regular operators, Acta Sci. Math. (Szeged) 15 (1953), 38-56. MR 15, 134.

3. N. Dunford and J. T. Schwartz, Linear operators. I: General theory, Pure and Appl. Math., vol. 7, Interscience, New York, 1958. MR 22 \#302. CANADA

DEPARTMENT OF MATHEMATICS, QUEEN'S UNIVERSITY, KINGSTON, ONTARIO, 